\title{
STATIONARY PROBLEMS WITH NONLOCAL BOUNDARY CONDITIONS
}

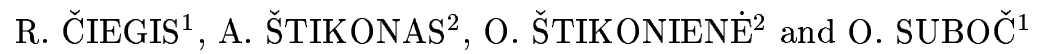 \\ ${ }^{1}$ Vilnius Gediminas Technical University, \\ ${ }^{2}$ Institute of Mathematics and Informatics \\ ${ }^{1}$ Saulètekio 11, Vilnius, Lithuania, ${ }^{2}$ Akademijos 4, 2600, Vilnius, Lithuania \\ E-mail: rc@fm.vtu.lt, Arturas.Stikonas@fm.vtu.lt, \\ E-mail: olgast@ktl.mii.lt, os@fm.vtu.lt
}

Received October 1, 2001

\begin{abstract}
In this article a stationary problems with general nonlocal boundary conditions is considered. The differential problems and finite difference schemes for solving this problem are investigated. Stability estimates are proved in the maximum norm and the non-negativity of the solution is investigated. All theoretical results are illustrated by representative examples.
\end{abstract}

\section{INTRODUCTION}

During the last decade there has been a growing interest in the development of numerical methods for solving problems with nonlocal boundary conditions $[2 ; 3 ; 4 ; 8 ; 9]$. Let consider the parabolic equation

$$
\frac{\partial u}{\partial t}=\frac{\partial}{\partial x}\left(p(x, t) \frac{\partial u}{\partial x}\right)-q(x, t) u+f(x, t, u), x \in(0,1), t \in(0, T]
$$

with the nonlocal boundary conditions

$$
\begin{aligned}
& u(0, t)=\gamma_{0}\left(\alpha_{0}(t) u\left(a_{0}(t), t\right)+\int_{0}^{1} \beta_{0}(x, t) u(x, t) \mathrm{d} x\right)+f_{0}(t), t \in(0, T] \\
& u(1, t)=\gamma_{1}\left(\alpha_{1}(t) u\left(a_{1}(t), t\right)+\int_{0}^{1} \beta_{1}(x, t) u(x, t) \mathrm{d} x\right)+f_{1}(t), t \in(0, T]
\end{aligned}
$$


and the initial condition

$$
u(x, 0)=u_{0}(x), x \in[0,1]
$$

We assume that $p(x, t) \geqslant p_{0}>0,0 \leqslant a_{i}(t) \leqslant 1, q(x, t) \geqslant 0, \gamma=\left(\gamma_{0}, \gamma_{1}\right) \in$ $\mathbb{R}_{+}^{2}=\left\{\left(\gamma_{0}, \gamma_{1}\right) \mid \gamma_{0} \geqslant 0, \gamma_{1} \geqslant 0\right\}$ and kernels $\alpha_{0}, \alpha_{1}, \beta_{0}, \beta_{1}$ are known functions.

First nonlocal boundary conditions were formulated in the paper of Samarskii and Bitsadze [1]. They investigated an elliptic problem in the plane with the nonlocal boundary condition for a segment on the boundary. For the linear case of (1.1) with $p(x, t) \equiv 1, \alpha_{0}=\alpha_{1}=0$, Ekolin [2] proved the convergence of the forward and backward Euler methods. In these schemes, the integrals in the nonlocal boundary conditions are approximated by the trapezoidal rule. The convergence of these methods is proved under the assumption that

$$
\gamma_{0}\left(\left|\beta_{0}\right|, 1\right)<1, \quad \gamma_{1}\left(\left|\beta_{1}\right|, 1\right)<1,
$$

where $(\cdot, \cdot)$ denotes the standard inner product $(f, g)=\int_{0}^{1} f(x) g(x) \mathrm{d} x$. For the Crank-Nicolson method the convergence is proved under the assumption that

$$
\gamma_{0}\left\|\beta_{0}\right\|+\gamma_{1}\left\|\beta_{1}\right\|<\frac{\sqrt{3}}{2}
$$

where $\|f\|:=\sqrt{(f, f)}$. Using Galerkin method Fairweather and Lopez-Marcos [3] solved semi-linear parabolic problem with integral boundary conditions $\alpha_{0}=\alpha_{1}=0$ under the assumptions

$$
\gamma_{0}\left\|\beta_{0}\right\|<1, \quad \gamma_{1}\left\|\beta_{1}\right\|<1 .
$$

A domain $\Omega_{E}$ is defined by inequalities (1.5) or (1.7) and a domain $\Omega_{F}$ is defined by inequality (1.6) are represented in Fig. 1 for $\beta_{0}=\beta_{1} \equiv 1$.

Our final aim is to estimate the solution of the parabolic problem by solutions of appropriate stationary elliptic problems. This analysis will be presented in a separate article.

The purpose of this paper is to analyze differential and finite difference problems for stationary problems with nonlocal boundary conditions. We investigate how the existence of its solution depends on parameters $\gamma_{0}$ and $\gamma_{1}$. We find the domains $\Omega$ and $\Omega_{+}$in the $\mathbb{R}_{+}^{2}$, where the unique solution exists and this solution is nonnegative for nonnegative $f_{0}, f_{1}$, respectively. Further we investigate the stability of the solution in the maximum norm with respect to values $f_{0}$ and $f_{1}$. 


\section{DIFFERENTIAL STATIONARY PROBLEM}

The stationary problem corresponding to parabolic problem (1.1)-(1.4) is given by

$$
\begin{aligned}
& l(u):=-\frac{\mathrm{d}}{\mathrm{d} x}\left(p(x) \frac{\mathrm{d} u}{\mathrm{~d} x}\right)+q(x) u=0, x \in(0,1), \\
& u(0)=\gamma_{0}\left(\alpha_{0} u\left(a_{0}\right)+\int_{0}^{1} \beta_{0}(x) u(x) \mathrm{d} x\right)+f_{0}, a_{0} \in[0,1], \\
& u(1)=\gamma_{1}\left(\alpha_{1} u\left(a_{1}\right)+\int_{0}^{1} \beta_{1}(x) u(x) \mathrm{d} x\right)+f_{1}, a_{1} \in[0,1] .
\end{aligned}
$$

Let $u$ be a classical solution, i.e. $u \in C^{2}(0,1) \cap C^{1}[0,1]$ for $p \in C^{1}[0,1]$, $q \in C[0,1]$ and kernels $\beta_{i} \in L_{1}(0,1)$. We denote $\|\cdot\|_{q}=\left(\int_{0}^{1}|\cdot|{ }^{q} \mathrm{~d} x\right)^{1 / q}$ the norm in the space $L_{q}(0,1)$ for $q \in[1, \infty)$ and $\|\cdot\|_{\infty}=\operatorname{vrai} \sup _{x \in(0,1)}|\cdot|$ the norm in the space $L_{\infty}(0,1)$. For continuous functions $u$ the norm $\|u\|_{\infty}=\|u\|_{C}$.

Let us define linear functionals:

$$
\begin{aligned}
& <k_{0}, u>:=\alpha_{0} u\left(a_{0}\right)+\int_{0}^{1} \beta_{0}(x) u(x) \mathrm{d} x, \\
& <k_{1}, u>:=\alpha_{1} u\left(a_{1}\right)+\int_{0}^{1} \beta_{1}(x) u(x) \mathrm{d} x .
\end{aligned}
$$

So we rewrite the problem (2.1)-(2.3) in the following way

$$
\left\{\begin{array}{l}
l(u)=0 \\
u(0)=\gamma_{0}<k_{0}, u>+f_{0} \\
u(1)=\gamma_{1}<k_{1}, u>+f_{1}
\end{array}\right.
$$

Proposition 2.1. If $u_{1}$ and $u_{2}$ are solutions of boundary value problems

$$
\left\{\begin{array} { r } 
{ - \frac { \mathrm { d } } { \mathrm { d } x } ( p ( x ) \frac { \mathrm { d } u _ { 1 } } { \mathrm { d } x } ) + q _ { 1 } ( x ) u _ { 1 } = 0 , } \\
{ u _ { 1 } ( 0 ) = f _ { 0 } , } \\
{ u _ { 1 } ( 1 ) = f _ { 1 } ; }
\end{array} \quad \left\{\begin{array}{r}
-\frac{\mathrm{d}}{\mathrm{d} x}\left(p(x) \frac{\mathrm{d} u_{2}}{\mathrm{~d} x}\right)+q_{2}(x) u_{2}=0, \\
u_{2}(0)=g_{0}, \\
u_{2}(1)=g_{1},
\end{array}\right.\right.
$$

respectively, and $0 \leqslant q_{1}(x) \leqslant q_{2}(x), 0 \leqslant g_{j} \leqslant f_{j}, j=0,1$, then

$$
0 \leqslant u_{2}(x) \leqslant u_{1}(x) .
$$

Proof. We get estimates $u_{j} \geqslant 0, j=0,1$, from the maximum principle. The function $u(x)=u_{1}(x)-u_{2}(x)$ satisfies the problem:

$$
\left\{\begin{array}{r}
-\frac{\mathrm{d}}{\mathrm{d} x}\left(p(x) \frac{\mathrm{d} u}{\mathrm{~d} x}\right)+q_{1}(x) u=\left(q_{2}(x)-q_{1}(x)\right) u_{2}, \\
u(0)=f_{0}-g_{0}, \quad u(1)=f_{1}-g_{1} .
\end{array}\right.
$$



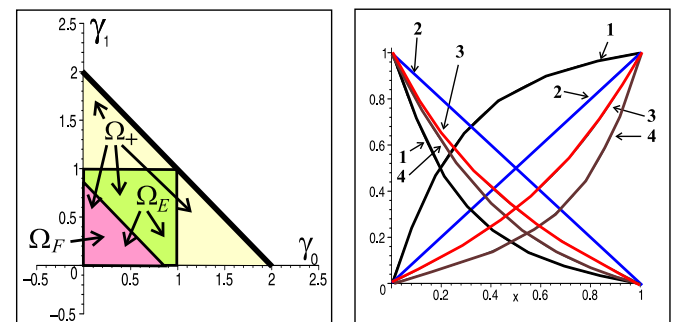

Figure 1. The domains $\Omega_{E}, \Omega_{F}$ and $\Omega_{+}$.

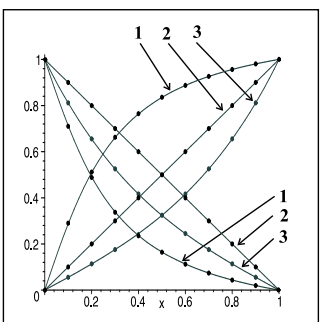

Figure 3. The functions $\Phi_{0}$ and $\Phi_{1}$.

Since $\left(q_{2}(x)-q_{1}(x)\right) u_{1} \geqslant 0, f_{0}-g_{0} \geqslant 0, f_{1}-g_{1} \geqslant 0$, then the inequality $u \geqslant 0$ follows from the maximum principle.

In order to investigate the nonlocal problem we will modify the method, which was proposed in [9]. Let solve two auxiliary problems with classical boundary conditions:

$$
\text { a) }\left\{\begin{array} { l } 
{ l ( \varphi _ { 0 } ) = 0 , } \\
{ \varphi _ { 0 } ( 0 ) = 1 , } \\
{ \varphi _ { 0 } ( 1 ) = 0 ; }
\end{array} \quad \text { b) } \left\{\begin{array}{l}
l\left(\varphi_{1}\right)=0, \\
\varphi_{1}(0)=0, \\
\varphi_{1}(1)=1 .
\end{array}\right.\right.
$$

We get estimates $0 \leqslant \varphi_{j}(x) \leqslant 1, x \in[0,1](j=0,1)$ from the maximum principle for elliptic problems. From this principle follows that $\varphi_{0}$ is non increasing function and $\varphi_{1}$ is non decreasing function. From Proposition 2.1 we obtain $0 \leqslant \varphi_{0}(x)+\varphi_{1}(x) \leqslant 1$.

Next we present some typical examples of functions $\varphi_{i}$ (see Fig. 2).

Example 2.1. Let coefficient $q \equiv 0$. It is easy to find solutions

$$
\varphi_{0}(x)=\frac{\int_{x}^{1} p^{-1}(s) \mathrm{d} s}{\int_{0}^{1} p^{-1}(s) \mathrm{d} s}, \quad \varphi_{1}(x)=\frac{\int_{0}^{x} p^{-1}(s) \mathrm{d} s}{\int_{0}^{1} p^{-1}(s) \mathrm{d} s} .
$$

For $p(x)=1+p_{0} x^{2}$ we have functions (curves 1 in Fig. 2 are obtained for $\left.p_{0}=16\right)$

$$
\varphi_{0}=1-\frac{\arctan \left(\sqrt{p_{0} x}\right)}{\arctan \left(\sqrt{p_{0}}\right)}, \quad \varphi_{1}=\frac{\arctan \left(\sqrt{p_{0} x}\right)}{\arctan \left(\sqrt{p_{0}}\right)} .
$$

Example 2.2. Taking $p \equiv 1$ and $q \equiv 0$ we obtain functions (curves 2)

$$
\varphi_{0}(x)=1-x, \quad \varphi_{1}(x)=x .
$$

Example 2.3. For constant coefficients $p \equiv 1, q \equiv q_{0}$ we obtain the following functions (curves 3 for $q_{0}=4$ )

$$
\varphi_{0}(x)=\frac{\sinh \left(\sqrt{q_{0}}(1-x)\right)}{\sinh \sqrt{q_{0}}}, \quad \varphi_{1}(x)=\frac{\sinh \left(\sqrt{q_{0}} x\right)}{\sinh \sqrt{q_{0}}} .
$$


Example 2.4. For variable coefficient $q(x)=q_{0}\left(1+q_{0} x^{2}\right)$ and $p \equiv 1$, we obtain (curves 4 for $q_{0}=4$ )

$$
\varphi_{0}(x)=\mathrm{e}^{q_{0} x^{2} / 2}\left(1-\frac{\operatorname{erf}\left(\sqrt{q_{0}} x\right)}{\operatorname{erf}\left(\sqrt{q_{0}}\right)}\right), \quad \varphi_{1}(x)=\mathrm{e}^{q_{0}\left(x^{2}-1\right) / 2} \frac{\operatorname{erf}\left(\sqrt{q_{0}} x\right)}{\operatorname{erf}\left(\sqrt{q_{0}}\right)}
$$

We write the solution of problem (2.6) in the form

$$
u=\varphi_{0}(x) y_{0}+\varphi_{1}(x) y_{1}
$$

Equation $l(u)=0$ holds trivially. The boundary conditions provide the linear algebraic system

$$
\left\{\begin{array}{l}
y_{0}=\gamma_{0}<k_{0}, \varphi_{0}>y_{0}+\gamma_{0}<k_{0}, \varphi_{1}>y_{1}+f_{0} \\
y_{1}=\gamma_{1}<k_{1}, \varphi_{0}>y_{0}+\gamma_{1}<k_{1}, \varphi_{1}>y_{1}+f_{1}
\end{array}\right.
$$

Let define the unitary matrix $\mathbf{I}$ and matrices

$$
\begin{aligned}
\mathbf{K}_{0} & :=\left(\begin{array}{ll}
k_{00} & k_{01} \\
k_{10} & k_{11}
\end{array}\right)=\left(\begin{array}{ll}
<k_{0}, \varphi_{0}> & <k_{0}, \varphi_{1}> \\
<k_{1}, \varphi_{0}> & <k_{1}, \varphi_{1}>
\end{array}\right), \mathbf{G}:=\left(\begin{array}{cc}
\gamma_{0} & 0 \\
0 & \gamma_{1}
\end{array}\right), \\
\Phi_{0}(x) & :=\left(\varphi_{0}(x) \varphi_{1}(x)\right), \mathbf{Y}:=\left(\begin{array}{l}
y_{0} \\
y_{1}
\end{array}\right), \quad \mathbf{F}:=\left(\begin{array}{l}
f_{0} \\
f_{1}
\end{array}\right) .
\end{aligned}
$$

Then we rewrite system (2.9) in the matrix form

$$
\mathbf{A}_{0} \mathbf{Y}=\mathbf{F},
$$

where $\mathbf{A}_{0}:=\mathbf{I}-\mathbf{G K}_{0}$ and obtain

$$
u=\Phi_{0}(x) \mathbf{Y} .
$$

Example 2.5. Let take the following coefficients $\alpha_{0}=\alpha_{1}=1, \beta_{0}=\beta_{1} \equiv$ $0, a_{0}=a \in[0,1], a_{1}=b \in[0,1]$, and $p \equiv 1, q \equiv 0$. Then we get matrices

$$
\mathbf{K}_{0}=\left(\begin{array}{cc}
1-a & a \\
1-b & b
\end{array}\right), \mathbf{A}_{0}=\left(\begin{array}{cc}
1-\gamma_{0}(1-a) & -\gamma_{0} a \\
-\gamma_{1}(1-b) & 1-\gamma_{1} b
\end{array}\right)
$$

Example 2.6. Let take the following coefficients $\alpha_{0}=\alpha_{1}=0, \beta_{0}=6(a-x)$, $\beta_{1}=12(x-b), a, b \in[0,1]$, and $p \equiv 1, q \equiv 0$. Then we get matrices

$$
\mathbf{K}_{0}=\left(\begin{array}{cc}
-1+3 a & -2+3 a \\
2-6 b & 4-6 b
\end{array}\right), \mathbf{A}_{0}=\left(\begin{array}{cc}
1-\gamma_{0}(-1+3 a) & -\gamma_{0}(-2+3 a) \\
-\gamma_{1}(2-6 b) & 1-\gamma_{1}(4-6 b)
\end{array}\right)
$$


This example illustrates the case when kernels aren't nonnegative and matrix $\mathbf{K}_{0}$ can have negative elements. For $a=\frac{1}{6}, \frac{1}{2}, \frac{5}{8}, \frac{3}{4}$ and $b=\frac{1}{2}$ we obtain matrices

$$
\mathbf{K}_{0}=\left(\begin{array}{cc}
-\frac{1}{2} & -\frac{3}{2} \\
-1 & 1
\end{array}\right),\left(\begin{array}{cc}
\frac{1}{2} & -\frac{1}{2} \\
-1 & 1
\end{array}\right),\left(\begin{array}{cc}
\frac{7}{8} & -\frac{1}{8} \\
-1 & 1
\end{array}\right),\left(\begin{array}{cc}
\frac{5}{4} & \frac{1}{4} \\
-1 & 1
\end{array}\right)
$$

We will investigate solvability of system (2.10) and properties of its solution (2.11) in Sec. 4.

\section{DISCRETE PROBLEM}

In the domain $[0,1]$ we introduce meshes

$$
\bar{\omega}^{h}=\left\{0=x_{0}<x_{1}<\cdots<x_{n}=1\right\}, \quad \omega^{h}=\bar{\omega}^{h} \backslash\left\{x_{0}, x_{n}\right\}
$$

with step sizes $h_{i}=x_{i}-x_{i-1}, 1 \leqslant i \leqslant n, h_{0}=h_{n+1}=0$, and semi-integer mesh

$$
\omega_{1 / 2}^{h}=\left\{x_{i+\frac{1}{2}} \mid x_{i+\frac{1}{2}}=\left(x_{i}+x_{i+1}\right) / 2,0 \leqslant i \leqslant n-1\right\}
$$

with step sizes $h_{i+\frac{1}{2}}=\left(h_{i}+h_{i+1}\right) / 2,0 \leqslant i \leqslant n$. A mesh $\omega$ will denote one of the meshes $\bar{\omega}^{h}, \omega^{h}, \bar{\omega}_{1 / 2}^{h}$. We denote $h=\max _{1 \leqslant i \leqslant n} h_{i}$. Let $\mathcal{D}(\omega)$ be a space of real valued functions which are defined on the mesh $\omega$ and $\mathcal{D}[0,1]$ is a space of real valued functions on $[0,1]$.

We define the following mesh operators

$$
(\delta Z)_{i+\frac{1}{2}}=\frac{Z_{i+1}-Z_{i}}{h_{i+1}}, Z \in \mathcal{D}\left(\bar{\omega}^{h}\right), \quad(\delta Z)_{i}=\frac{Z_{i+\frac{1}{2}}-Z_{i-\frac{1}{2}}}{h_{i+\frac{1}{2}}}, Z \in \mathcal{D}\left(\omega_{1 / 2}^{h}\right) .
$$

We introduce the following mesh norms:

$$
\|Z\|_{1, \bar{\omega}^{h}}:=\sum_{i=0}^{n}\left|Z_{i}\right| h_{i+\frac{1}{2}}, Z \in \mathcal{D}\left(\bar{\omega}^{h}\right), \quad\|Z\|_{\infty, \omega}:=\max _{x \in \omega}|Z(x)|, Z \in \mathcal{D}(\omega) .
$$

Remark 3.1. In this section we always use capital letters (e.g. $U, V$ ) for functions defined on mesh $\omega$ and letters (e.g. $u, v$ ) for functions defined on interval $[0,1]$. Further for simplification of notation we omit subscripts $\omega$ or $[0,1]$ in norms and inner product. In this case we can use the same notation for continuous and discrete norms, inner products and linear functionals, e.g.:

$$
(u, v):=\int_{0}^{1} u(x) v(x) \mathrm{d} x, \quad(U, V):=\sum_{i=0}^{n} U_{i} V_{i} h_{i+\frac{1}{2}},
$$


where $u, v \in \mathcal{D}[0,1], U, V \in \mathcal{D}\left(\bar{\omega}^{h}\right)$.

Let $\widetilde{Z}$ be a linear interpolant

$$
\widetilde{Z}(x)=\frac{x_{i}-x}{h_{i}} Z_{i-1}+\frac{x-x_{i-1}}{h_{i}} Z_{i} \text { for } x \in\left[x_{i-1}, x_{i}\right] .
$$

We approximate problem (2.1)-(2.3) by the following finite-difference scheme:

$$
\begin{aligned}
L(U) & :=-\delta(P \delta U)+Q U=0 \quad \text { in } \omega^{h}, \\
\left.U\right|_{i=0} & =\gamma_{0}\left(\alpha_{0} \widetilde{U}\left(a_{0}\right)+\left(B_{0}, U\right)\right)+f_{0}, \\
\left.U\right|_{i=n} & =\gamma_{1}\left(\alpha_{1} \widetilde{U}\left(a_{1}\right)+\left(B_{1}, U\right)\right)+f_{1},
\end{aligned}
$$

where $U, B_{0}, B_{1} \in \mathcal{D}\left(\bar{\omega}^{h}\right), 0<p_{0} \leqslant P \in \mathcal{D}\left(\omega_{1 / 2}^{h}\right), 0 \leqslant Q \in \mathcal{D}\left(\omega^{h}\right)$.

Lemma 3.1. Let coefficients $\beta_{0}, \beta_{1} \in C^{2}[0,1], p \in C^{3}[0,1], q \in C^{2}[0,1]$. Assume that problem (2.1)-(2.3) has a unique solution $u \in C^{4}[0,1]$. Let $h=$ const, $(\delta P)_{i}=p^{\prime}\left(x_{i}\right)+\mathcal{O}\left(h^{2}\right), \frac{1}{2}\left(P_{i+\frac{1}{2}}+P_{i-\frac{1}{2}}\right)=p\left(x_{i}\right)+\mathcal{O}\left(h^{2}\right), Q_{i}=q\left(x_{i}\right)+$ $\mathcal{O}\left(h^{2}\right)$. Then the truncation error for finite difference scheme (3.1)-(3.3) is $\psi=\mathcal{O}\left(h^{2}\right)$.

Proof. As well known [5] the truncation error for equation (3.1) is of order $\mathcal{O}\left(h^{2}\right)$. The integrals on the right hand side of expressions (2.2)-(2.3) are approximated by the trapezoidal rule, thus quadrature error is bounded by $C h^{2}$ for functions in space $C^{2}[0,1][6]$. Truncation error for linear interpolation is $\mathcal{O}\left(h^{2}\right)$. Then total truncation error is $\mathcal{O}\left(h^{2}\right)$.

Let us introduce linear functionals:

$$
\begin{aligned}
& <K_{0}, U>:=\alpha_{0} \widetilde{U}\left(a_{0}\right)+\left(B_{0}, U\right), \\
& <K_{1}, U>:=\alpha_{1} \widetilde{U}\left(a_{1}\right)+\left(B_{1}, U\right) .
\end{aligned}
$$

Then we rewrite problem (3.1)-(3.3) in the following form

$$
\left\{\begin{array}{l}
L(U)=0 \\
\left.U\right|_{i=0}=\gamma_{0}<K_{0}, U>+f_{0}, \\
\left.U\right|_{i=n}=\gamma_{1}<K_{1}, U>+f_{1} .
\end{array}\right.
$$

Proposition 3.1. If $U_{1}$ and $U_{2}$ are solutions of problems

$$
\left\{\begin{array} { r } 
{ - \delta ( P \delta U _ { 1 } ) + Q _ { 1 } U _ { 1 } = 0 , } \\
{ U _ { 1 } | _ { i = 0 } = f _ { 0 } , } \\
{ U _ { 1 } | _ { i = n } = f _ { 1 } ; }
\end{array} \quad \left\{\begin{array}{r}
-\delta\left(P \delta U_{2}\right)+Q_{2} U_{2}=0, \\
\left.U_{2}\right|_{i=0}=g_{0}, \\
\left.U_{2}\right|_{i=n}=g_{1}
\end{array}\right.\right.
$$

and $0 \leqslant Q_{1}\left(x_{i}\right) \leqslant Q_{2}\left(x_{i}\right), 0 \leqslant g_{j} \leqslant f_{j}, j=0,1$, then $0 \leqslant U_{2}\left(x_{i}\right) \leqslant U_{1}\left(x_{i}\right)$. 
Analogously to the differential case we solve two auxiliary discrete boundary problems:

$$
\text { A) }\left\{\begin{array}{l}
L\left(\Phi_{0}\right)=0, \\
\left.\Phi_{0}\right|_{i=0}=1, \\
\left.\Phi_{0}\right|_{i=n}=0 ;
\end{array} \quad B\right)\left\{\begin{array}{l}
L\left(\Phi_{1}\right)=0 \\
\left.\Phi_{1}\right|_{i=0}=0 \\
\left.\Phi_{1}\right|_{i=n}=1
\end{array}\right.
$$

The estimates $0 \leqslant \Phi_{j}\left(x_{i}\right) \leqslant 1, x_{i} \in \bar{\omega}^{h}(j=0,1)$ and monotonicity of these solutions follow from the discrete maximum principle [5]. From Proposition 3.1 we get $0 \leqslant \Phi_{0}\left(x_{i}\right)+\Phi_{1}\left(x_{i}\right) \leqslant 1$.

As examples we consider finite difference approximations of three problems in Sec. 2. The graphics of discrete solutions are presented in Fig. 3.

Example 3.1. If $Q=0$ then we obtain

$$
\Phi_{0}\left(x_{i}\right)=\frac{\sum_{k=i}^{n} h_{k} / P_{k-\frac{1}{2}}}{\sum_{k=1}^{n} h_{k} / P_{k-\frac{1}{2}}}, \quad \Phi_{1}\left(x_{i}\right)=\frac{\sum_{k=0}^{i} h_{k} / P_{k-\frac{1}{2}}}{\sum_{k=1}^{n} h_{k} / P_{k-\frac{1}{2}}} .
$$

Example 3.2. Let coefficients be $P \equiv 1, Q \equiv 0$. Then we obtain

$$
\Phi_{0}\left(x_{i}\right)=1-x_{i}, \quad \Phi_{1}\left(x_{i}\right)=x_{i} .
$$

Example 3.3. Let coefficients be $P \equiv 1, Q \equiv q_{0}$ and $h=1 / n=$ const. Then we obtain

$$
\Phi_{0}=\frac{\lambda_{2}^{n} \lambda_{1}^{i}-\lambda_{1}^{n} \lambda_{2}^{i}}{\lambda_{2}^{n}-\lambda_{1}^{n}}, \quad \Phi_{1}=\frac{\lambda_{2}^{i}-\lambda_{1}^{i}}{\lambda_{2}^{n}-\lambda_{1}^{n}}
$$

where $\lambda_{1,2}=\left(2+q_{0} h^{2} \mp \sqrt{q_{0} h^{2}\left(4+q_{0} h^{2}\right)}\right) / 2$.

We search the solution of finite-difference scheme (3.6) in the form

$$
U=\Phi_{0}\left(x_{i}\right) y_{0}+\Phi_{1}\left(x_{i}\right) y_{1}
$$

The boundary conditions provide linear algebraic system

$$
\left\{\begin{array}{l}
y_{0}=\gamma_{0}<K_{0}, \Phi_{0}>y_{0}+\gamma_{0}<K_{0}, \Phi_{1}>y_{1}+f_{0} \\
y_{1}=\gamma_{1}<K_{1}, \Phi_{0}>y_{0}+\gamma_{1}<K_{1}, \Phi_{1}>y_{1}+f_{1}
\end{array}\right.
$$

Let denote matrices

$$
\mathbf{K}_{h}:=\left(\begin{array}{cc}
<K_{0}, \Phi_{0}> & <K_{0}, \Phi_{1}> \\
<K_{1}, \Phi_{0}> & <K_{1}, \Phi_{1}>
\end{array}\right), \quad \Phi_{h}\left(x_{i}\right):=\left(\Phi_{0}\left(x_{i}\right) \Phi_{1}\left(x_{i}\right)\right) .
$$


Then we rewrite the system (3.9) in the matrix form

$$
\mathbf{A}_{h} \mathbf{Y}=\mathbf{F},
$$

where $\mathbf{A}_{h}:=\mathbf{I}-\mathbf{G K}_{h}$ and obtain

$$
U=\Phi_{h}(x) \mathbf{Y}
$$

Example 3.4. Let coefficients be $\alpha_{0}=0, \alpha_{1}=1, a_{1}=a, \beta_{0}=1, \beta_{1}=0$, $P=1, Q=0$ and $h=$ const. Then

$$
\mathbf{K}_{h}=\left(\begin{array}{cc}
1 / 2 & 1 / 2 \\
1-a & a
\end{array}\right), \mathbf{A}_{h}=\left(\begin{array}{cc}
1-\gamma_{0} / 2 & -\gamma_{0} / 2 \\
-\gamma_{1}(1-a) & 1-\gamma_{1} a
\end{array}\right)
$$

\section{ALGEBRAIC CONDITIONS FOR EXISTENCE AND UNIQUENESS OF SOLUTION}

In both differential and difference cases we get that solution of nonlocal problem is given by (see (2.11) and (3.11))

$$
v(x)=\mathbf{\Psi}(x) \mathbf{Y}, \quad \mathbf{\Psi}(x):=\left(\Psi_{0}(x) \Psi_{1}(x)\right),
$$

where $x \in[0,1]$ or $x \in \bar{\omega}^{h}, \Psi_{0}(x) \geqslant 0, \Psi_{1}(x) \geqslant 0, \Psi_{0}(x)+\Psi_{1}(x) \leqslant 1$, and vector $\mathbf{Y}$ satisfies a linear algebraic system (see (2.10) and (3.10))

$$
\mathbf{A Y}=\mathbf{F},
$$

where $\mathbf{A}:=\mathbf{I}-\mathbf{G K}$ and

$$
\mathbf{A}=\left(\begin{array}{ll}
a_{00} & a_{01} \\
a_{10} & a_{11}
\end{array}\right), \mathbf{K}=\left(\begin{array}{ll}
k_{00} & k_{01} \\
k_{10} & k_{11}
\end{array}\right)=\left(\begin{array}{cc}
<\varkappa_{0}, \Psi_{0}> & <\varkappa_{0}, \Psi_{1}> \\
<\varkappa_{1}, \Psi_{0}> & <\varkappa_{1}, \Psi_{1}>
\end{array}\right) .
$$

In this section we investigate the solvability of problem (4.1)-(4.2) and obtain conditions, which guarantee that this solution is nonnegative. All results are formulated in terms of matrix A. Let us use standard vector and matrix norms

$$
\begin{array}{rlrl}
\|\mathbf{Y}\|_{\infty} & :=\max \left\{\left|y_{0}\right|,\left|y_{1}\right|\right\}, \quad\|\mathbf{A}\|_{\infty}: & :=\max \left\{\left|a_{00}\right|+\left|a_{01}\right|,\left|a_{10}\right|+\left|a_{11}\right|\right\} \\
\|\mathbf{Y}\|_{1} & :=\left|y_{0}\right|+\left|y_{1}\right|, & \|\mathbf{A}\|_{1} & :=\max \left\{\left|a_{00}\right|+\left|a_{10}\right|,\left|a_{01}\right|+\left|a_{11}\right|\right\} .
\end{array}
$$

Then we have estimates

$$
\begin{aligned}
\|\mathbf{\Psi}(x)\|_{\infty} & =\max _{j=0,1}\left\{\left|\Psi_{j}(x)\right|\right\} \leqslant 1, \quad\|\mathbf{\Psi}(x)\|_{1}=\Psi_{0}(x)+\Psi_{1}(x) \leqslant 1, \\
\|\mathbf{K}\|_{\infty} & =\max _{j=0,1}\left\{\left|<\varkappa_{j}, \Psi_{0}>\right|+\left|<\varkappa_{j}, \Psi_{1}>\right|\right\} \leqslant \max _{j=0,1}\left\{<\left|\varkappa_{j}\right|, 1>\right\}, \\
\|\mathbf{K}\|_{1} & =\max _{j=0,1}\left\{\left|<\varkappa_{0}, \Psi_{j}>\right|+\left|<\varkappa_{1}, \Psi_{j}>\right|\right\} \leqslant<\left|\varkappa_{0}\right|, 1>+<\left|\varkappa_{1}\right|, 1>,
\end{aligned}
$$


where we get linear functional $\langle|\varkappa|, \Psi\rangle$ from the functional $\langle\varkappa, \Psi\rangle$ by using a module of the kernel.

Lemma 4.1. Let matrix $\mathbf{A}$ satisfies the condition $\operatorname{det} \mathbf{A} \neq 0$. Then there exists a unique solution of nonlocal problem and

$$
\|v\|_{\infty} \leqslant \frac{\|\mathbf{A}\|_{1}}{|\operatorname{det} \mathbf{A}|}\|\mathbf{F}\|_{\infty}, \quad\|v\|_{\infty} \leqslant \frac{\|\mathbf{A}\|_{\infty}}{|\operatorname{det} \mathbf{A}|}\|\mathbf{F}\|_{1} .
$$

Proof. For system (4.2) a unique solution exists if $\operatorname{det} \mathbf{A} \neq 0$ and in this case

$$
\mathbf{Y}=\mathbf{A}^{-1} \mathbf{F}, \text { where } \mathbf{A}^{-1}=\frac{1}{\operatorname{det} \mathbf{A}}\left(\begin{array}{cc}
a_{11} & -a_{01} \\
-a_{10} & a_{00}
\end{array}\right)
$$

Then we obtain the solution of nonlocal problem in the form

$$
v(x)=\mathbf{\Psi}(x) \mathbf{A}^{-1} \mathbf{F} .
$$

Equalities (4.3) and (4.4) imply that

$$
\begin{aligned}
& |v(x)| \leqslant \Psi_{0}(x)\left|y_{0}\right|+\Psi_{1}(x)\left|y_{1}\right| \leqslant\left(\Psi_{0}(x)+\Psi_{1}(x)\right)\|\mathbf{Y}\|_{\infty} \leqslant\left\|\mathbf{A}^{-1}\right\|_{\infty}\|\mathbf{F}\|_{\infty}, \\
& |v(x)| \leqslant \Psi_{0}(x)\left|y_{0}\right|+\Psi_{1}(x)\left|y_{1}\right| \leqslant\|\mathbf{Y}\|_{1} \leqslant\left\|\mathbf{A}^{-1}\right\|_{1}\|\mathbf{F}\|_{1} .
\end{aligned}
$$

Then using equalities $\left\|\mathbf{A}^{-1}\right\|_{\infty}=\|\mathbf{A}\|_{1} /|\operatorname{det} \mathbf{A}|$ and $\left\|\mathbf{A}^{-1}\right\|_{1}=\|\mathbf{A}\|_{\infty} /|\operatorname{det} \mathbf{A}|$ we complete the proof.

Example 4.1. Let the coefficients $p \equiv 1, q \equiv 0, f_{0}=0, \alpha_{0}=0, \alpha_{1}=1, a_{1}=$ $b, \beta_{0}=0, \beta_{1}=0$. In this case boundary condition are the following

$$
u(0)=0, \quad u(1)=\gamma_{1} u(a)+f_{1}
$$

Then $\varphi_{0}=1-x, \varphi_{1}=x$ and $k_{00}=k_{10}=0, k_{10}=1-b, k_{11}=b$. Thus $\operatorname{det} \mathbf{A}=1-\gamma_{1} b$ and we have singularity when $\gamma_{1} b=1$. Then function $u=C x$ is a solution for all $C \in \mathbb{R}$ if $f_{1}=0$ and there is no solution if $f_{1} \neq 0$.

Lemma 4.2. Let $\operatorname{det} \mathbf{A} \neq 0$ and conditions

$$
\begin{aligned}
& a_{00}, a_{11} \geqslant 0, \quad a_{01}, a_{10} \leqslant 0 \quad \text { for } \quad \operatorname{det} \mathbf{A}>0, \\
& a_{00}, a_{11} \leqslant 0, \quad a_{01}, a_{10} \geqslant 0 \quad \text { for } \quad \operatorname{det} \mathbf{A}<0 \text {, }
\end{aligned}
$$

are satisfied, then the solution $v$ is nonnegative for nonnegative $f_{0}$ and $f_{1}$.

Proof. Functions $\Psi_{i}$ are nonnegative. Thus $y_{0}$ and $y_{1}$ must be nonnegative. We obtain such solutions for positive matrix $\mathbf{A}^{-1}$, i.e. $\frac{a_{00}}{\operatorname{det} \mathbf{A}}, \frac{-a_{01}}{\operatorname{det} \mathbf{A}}, \frac{-a_{10}}{\operatorname{det} \mathbf{A}}$, $\frac{a_{11}}{\operatorname{det} \mathbf{A}} \geqslant 0$. 


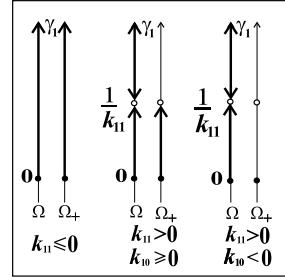

a)

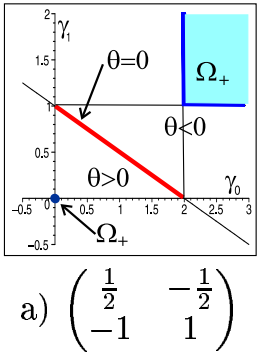

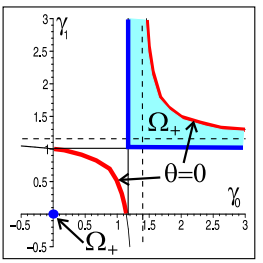

b) $\left(\begin{array}{cc}\frac{7}{8} & -\frac{1}{8} \\ -1 & 1\end{array}\right)$

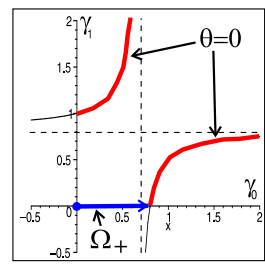

c) $\left(\begin{array}{cc}\frac{5}{4} & \frac{1}{4} \\ -1 & 1\end{array}\right)$

Figure 4. The do- Figure 5. The singular curve $\theta=0$ and domain $\Omega_{+}$for mains $\Omega$ and $\Omega_{+}$for matrices $\mathbf{K}$ with negative elements. one nonlocal condition.

\section{ANALYSIS OF SOLVABILITY CONDITIONS}

In this section we study the dependence of problem (4.1)-(4.2) solvability on parameters $\gamma_{0}$ and $\gamma_{1}$. The main existence condition $\operatorname{det} \mathbf{A} \neq 0$ can be written in the form

$$
\theta:=1-\gamma_{0} k_{00}-\gamma_{1} k_{11}+\gamma_{0} \gamma_{1} \operatorname{det} \mathbf{K} \neq 0 .
$$

We have a unique solution if $\gamma \in \Omega:=\left\{\gamma \in \mathbb{R}_{+}^{2} \mid \theta(\gamma) \neq 0\right\}$.

Corollary 5.1. Let $\theta \neq 0$. Then there exists a unique solution of nonlocal problem and the estimate

$$
\|v\|_{\infty} \leqslant|\theta|^{-1}\left(1+2 \max \left\{\gamma_{0}, \gamma_{1}\right\}\right)\left(<\left|\varkappa_{0}\right|, 1>+<\left|\varkappa_{1}\right|, 1>\right)\|\mathbf{F}\|_{s} .
$$

holds, where $s=1, \infty$.

Proof. We proved in Sec.4 the estimate

$$
\left.\|K\|_{s} \leqslant<\left|\varkappa_{0}\right|, 1\right\rangle+<\left|\varkappa_{1}\right|, 1>, s=1, \infty .
$$

Now we estimate the norms of the matrix I - GK:

$$
\begin{aligned}
& \|\mathbf{I}-\mathbf{G K}\|_{\infty} \leqslant 1+\max \left\{\gamma_{0}, \gamma_{1}\right\}\|\mathbf{K}\|_{\infty}, \\
& \|\mathbf{I}-\mathbf{G K}\|_{1} \leqslant 1+\left(\gamma_{0}+\gamma_{1}\right)\|\mathbf{K}\|_{1}
\end{aligned}
$$

and using Lemma 4.1 we complete the proof.

Corollary 5.2. Let $\theta \neq 0$ and conditions

$$
\begin{array}{lll}
\gamma_{0} k_{00} \leqslant 1, & \gamma_{1} k_{11} \leqslant 1, \quad \gamma_{0} k_{01} \geqslant 0, \quad \gamma_{1} k_{10} \geqslant 0 & \text { for } \theta>0 \\
\gamma_{0} k_{00} \geqslant 1, & \gamma_{1} k_{11} \geqslant 1, \quad \gamma_{0} k_{01} \leqslant 0, & \gamma_{1} k_{10} \leqslant 0
\end{array}
$$




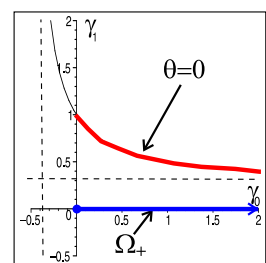

а) $\left(\begin{array}{cc}-\frac{1}{2} & -\frac{3}{2} \\ -1 & 1\end{array}\right)$

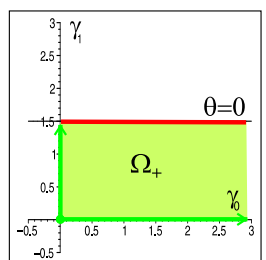

b) $\left(\begin{array}{ll}0 & 0 \\ \frac{2}{3} & \frac{1}{3}\end{array}\right)$

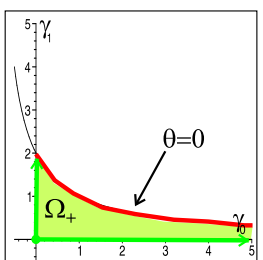

c) $\left(\begin{array}{ll}0 & 1 \\ \frac{2}{3} & \frac{1}{3}\end{array}\right)$

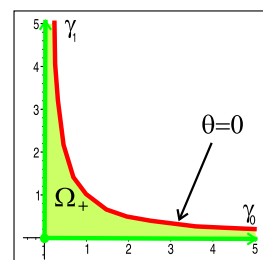

d) $\left(\begin{array}{ll}0 & 1 \\ 1 & 0\end{array}\right)$

Figure 6. The unbounded domains $\Omega_{+}$for matrices $\mathbf{K}$.

are satisfied. Then the solution $v$ is nonnegative for nonnegative $f_{0}$ and $f_{1}$.

Remark 5.1 [The case of one nonlocal condition]. Let consider the case $\varkappa_{0} \equiv 0\left(k_{00}=k_{01}=0\right)$. Then the unique solution exists for $\gamma_{1} k_{11} \neq 1$ and

$$
\Omega_{+}=\left\{\left(\gamma_{0}, \gamma_{1}\right) \mid \gamma_{1} k_{11}<1, \gamma_{1} k_{10} \geqslant 0\right\}
$$

(see Fig. 4). For problem with one nonlocal condition(without integral) the condition $\gamma_{1} k_{11} \neq 1$ is the same as in [9].

If $\gamma_{0}=0$ then we get the same condition (5.5), i.e. on the $\gamma_{1}$-axe(or $\gamma_{0}$-axe) we have the same situation as for one nonlocal condition.

The singularity points are on the hyperbola or on the line

$$
\begin{array}{r}
\left(\gamma_{0}-\frac{k_{11}}{\operatorname{det} \mathbf{K}}\right)\left(\gamma_{1}-\frac{k_{00}}{\operatorname{det} \mathbf{K}}\right)=\frac{k_{10} k_{01}}{(\operatorname{det} \mathbf{K})^{2}} \text { for } \operatorname{det} \mathbf{K} \neq 0, \\
\gamma_{0} k_{00}+\gamma_{1} k_{11}=1 \text { for } \operatorname{det} \mathbf{K}=0 .
\end{array}
$$

The hyperbola degenerate into two lines when $k_{00} k_{11}=0$.

Remark 5.2 [Domain $\Omega_{+}$for matrices with negative elements]. Such kernels and matrices we get in Example 2.6. If $k_{01}<0$ and $k_{10}<0$ then $\gamma_{0}=\gamma_{1}=0$ for $\theta>0$ and $\gamma_{0} k_{00} \geqslant 1, \gamma_{1} k_{11} \geqslant 1$ for $\theta<0$ (see Fig. 5a,b). If $k_{01} \geqslant 0$ and $k_{10}<0$ then $\gamma_{1}=0, \gamma_{0} k_{00}<1$ (see Fig. 5 c).

Remark 5.3 [Unbounded domains $\Omega_{+}$]. For some matrices the domain $\Omega_{+}$ is unbounded (see Fig. 6a). For nonnegative matrices (see Fig. 6b,c,d) the domain $\Omega_{+}$is unbounded if $k_{00}=0$ or $k_{11}=0$. In such cases the constant $1+2 \max \left\{\gamma_{0}, \gamma_{1}\right\}$ in the right hand of the inequality (5.2) is unbounded. Thus we must use more strong $\varepsilon$-condition and domain $\Omega_{+}^{\varepsilon}$ for $\gamma_{0}$ and $\gamma_{1}$ :

$$
\Omega_{+}^{\varepsilon}:=\left\{\gamma \in \Omega_{+}|| \theta \mid \geqslant \varepsilon>0, \gamma_{0} \leqslant \varepsilon^{-1}, \gamma_{1} \leqslant \varepsilon^{-1}\right\} .
$$




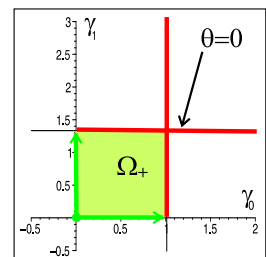

a) $\left(\begin{array}{cc}1 & 0 \\ \frac{1}{4} & \frac{3}{4}\end{array}\right)$

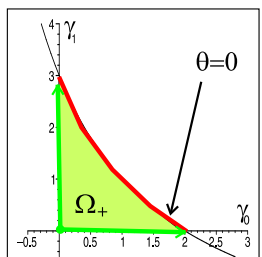

b) $\left(\begin{array}{ll}\frac{1}{2} & \frac{1}{2} \\ \frac{2}{3} & \frac{1}{3}\end{array}\right)$

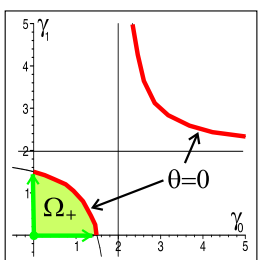

c) $\left(\begin{array}{ll}\frac{2}{3} & \frac{1}{3} \\ \frac{1}{3} & \frac{2}{3}\end{array}\right)$

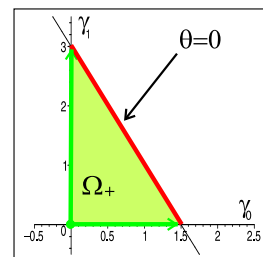

d) $\left(\begin{array}{ll}\frac{2}{3} & \frac{1}{3} \\ \frac{2}{3} & \frac{1}{3}\end{array}\right)$

Figure 7. The bounded domains $\Omega_{+}$for nonnegative matrices $\mathbf{K}$.
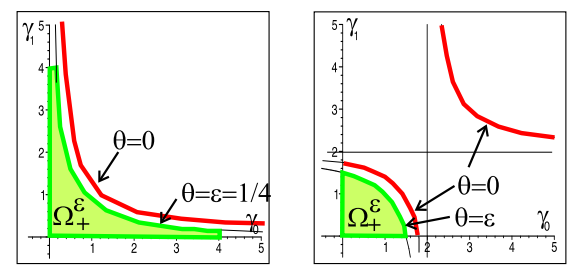

Figure 8. The domains $\Omega_{+}^{\varepsilon}$.
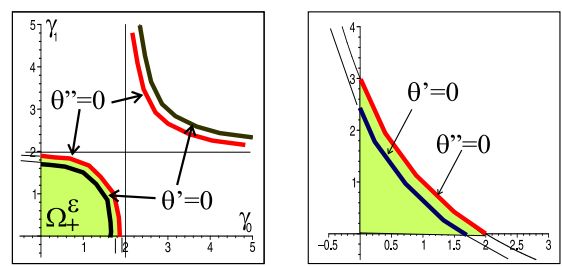

Figure 9. The domains $\Omega_{+}$for matrices $\mathbf{K}^{\prime} \geqslant \mathbf{K}^{\prime \prime} \geqslant \mathbf{0}$.

Lemma 5.1. Let $\gamma \in \Omega_{+}^{\varepsilon}$. Then there exists a unique solution $v$, and it is nonnegative for nonnegative $f_{0}, f_{1}$ and

$$
\|v\|_{\infty} \leqslant C\left(\varepsilon, \varkappa_{0}, \varkappa_{1}\right)\|\mathbf{F}\|_{s}, s=1, \infty
$$

where $C\left(\varepsilon, \varkappa_{0}, \varkappa_{1}\right):=\varepsilon^{-1}\left(1+2 \varepsilon^{-1}\right)\left(<\left|\varkappa_{0}\right|, 1\right\rangle+\left\langle\left|\varkappa_{1}\right|, 1>\right)$.

In many applications kernels for functional $\varkappa_{i}$ are nonnegative $\left(\alpha_{i} \geqslant 0, \beta_{i} \geqslant\right.$ $\left.0, B_{i} \geqslant 0\right)$. In this case the solution of problem (4.1)-(4.2) is nonnegative if $\theta>0$ (see Fig. 6b,c,d and Fig. 7) and the domain where $\theta>\varepsilon$ belongs to $\Omega_{+}$(see Fig. 8).

Lemma 5.2. Let $\theta \geqslant \varepsilon>0, \gamma_{0} \leqslant \varepsilon^{-1}, \gamma_{1} \leqslant \varepsilon^{-1}$. Then for nonnegative kernels and nonnegative $f_{0}$ and $f_{1}$ there exists a unique nonnegative solution of nonlocal problem (4.1)-(4.2) and the estimate (5.9) holds.

Remark 5.4. Let matrix $\mathbf{K}^{\prime} \geqslant \mathbf{K}^{\prime \prime} \geqslant \mathbf{0}$, i.e. $\Delta k_{i j}:=k_{i j}^{\prime}-k_{i j}^{\prime \prime} \geqslant 0$. Noting that for $\theta^{\prime} \geqslant 0$ the

$$
\begin{aligned}
\theta^{\prime \prime}-\theta^{\prime} & =\gamma_{0}\left(1-k_{11} \gamma_{1}\right) \Delta k_{00}+\gamma_{1}\left(1-k_{00} \gamma_{0}\right) \Delta k_{11} \\
& +\gamma_{0} \gamma_{1}\left(\Delta k_{00} \Delta k_{11}+k_{01} \Delta k_{10}+\Delta k_{01} k_{10}+\Delta k_{01} \Delta k_{10}\right) \geqslant 0
\end{aligned}
$$

we prove that domain $\Omega_{+}^{\prime \prime}$ is larger than $\Omega_{+}^{\prime}$ (see Fig. 9). If $q(x) \geqslant q_{0} \geqslant 0$ for the problem (2.1)-(2.3) (or $Q_{i} \geqslant q_{0} \geqslant 0$ for the problem (3.1)-(3.3)) 
then $\Psi_{j}^{q}(x) \geqslant \Psi_{j}^{q_{0}}(x) \geqslant \Psi_{j}^{0}(x), j=1,2$, and $k_{i j}^{q} \geqslant k_{i j}^{q_{0}} \geqslant k_{i j}^{0}, i, j=0,1$, i.e. $\Omega_{+}^{q} \subset \Omega_{+}^{q_{0}} \subset \Omega_{+}^{0}$.

In Fig. 1 we have illustrated the domains $\Omega_{E}$ and $\Omega_{F}$, that represented the solvability conditions under assumptions (1.5)-(1.7) in the case $\beta_{0}=\beta_{1} \equiv 1$. As we see in Fig. 1 the obtained domain $\Omega_{+}=\left\{\gamma \in \mathbb{R}_{+}^{2} \mid \gamma_{0}+\gamma_{1}<2\right\}$ is larger than $\Omega_{E}$ or $\Omega_{F}$.

\section{REFERENCES}

[1] A.Bitsadze and A.Samarskii. O nekotoryh prosteishih obobschenijah lineinyh ellipticheskih kraevyh zadach. Dokl. AN SSSR, 185 (4), 1969, 739-740.(in Russian)

[2] G.Ekolin. Finite difference methods for a nonlocal boundary value problem for the heat equation. BIT, 31, 1991, $245-261$.

[3] G.Fairweather and J.C.López-Marcos. Galerkin methods for a semilinear parabolic problem with nonlocal boundary conditions. Advances in Computational Mathematics, 6, $1996,243-262$.

[4] A.V.Goolin, N.I.Ionkin and V.A.Morozova. Difference schemes with nonlocal boundary conditions. Computational methods in applied mathematics, 1 (1), 2001, 62 - 71.

[5] A.Samarskii. The Theory of Difference Schemes. Marcel Dekker, Inc., New York Basel, 2001.

[6] A.Samarskii and A.Gulin. Stability of Difference Schemes. Nauka, Moscow, 1973. (in Russian)

[7] A.Samarskii and A.Gulin. Numerical Methods. Nauka, Moscow, 1989. (in Russian)

[8] M.Sapagovas. Kraevaja zadacha s nelokalnym usloviem dlja sistemy obyknovennyh differencialnyh uravnenii. Differents. Uravn., 36 (7), 2000, 971-978.(in Russian)

[9] M.Sapagovas and R.Čiegis. On some boundary problems with nonlocal conditions. Differents. Uravn., 23 (7), 1987, 1268-1274.(in Russian)

\section{Stacionarusis uždavinys su nelokoliomis kraštinėmis sąlygomis}

R. Čiegis, A. Štikonas, O. Štikonienè, O. Suboč

Straipsnyje nagrinejjamas uždavinys su nelokoliosiomis kraštinèmis sąlygomis, susiejančiomis sprendinio reikšmę srities kraštuose su sprendinio reikšme vidiniame taške ir/arba sprendinio integralu. Tiriamas diferencialinis uždavinys ir baigtinių skirtumų schema, aproksimuojanti ši uždavinįi. Gautos pakankamos sąlygos sprendinio egzistavimui ir vienačiai. Surastos sąlygos, kada sprendinys yra neneigiamas ir stabilus tolygioje normoje. Visi teoriniai rezultatai iliustruojami atitinkamais pavyzdžiais. 